\title{
CAR2, a prestalk cAMP receptor required for normal tip formation and late development of Dictyostelium discoideum
}

\author{
Charles L. Saxe III, ${ }^{1,3}$ Gail T. Ginsburg, ${ }^{1}$ John M. Louis, ${ }^{1}$ Ronald Johnson, ${ }^{2}$ Peter N. Devreotes, ${ }^{2}$ \\ and Alan R. Kimmel ${ }^{1,4}$ \\ ${ }^{1}$ Laboratory of Cellular and Developmental Biology, NIDDK (Bldg 6/B1-22), National Institutes of Health, Bethesda, \\ Maryland 20892, USA; ${ }^{2}$ Department of Biological Chemistry, Johns Hopkins University, School of Medicine, Baltimore, \\ Maryland 21205 USA
}

Extracellular CAMP serves as a primary signaling molecule to regulate the development of Dictyostelium discoideum. It is required for chemotaxis, aggregation, cytodifferentiation, and morphogenetic movement. The receptors for CAMP are members of the family of cell-surface receptors that are linked to G proteins and characterized by seven putative transmembrane domains. Previously, we have isolated the gene for the cAMP receptor subtype 1 (CAR1) from Dictyostelium and suggested that several genes related to CAR1 were present in the genome. Here, we describe a family of cAMP receptor genes of Dictyostelium and the isolation and function of the gene for the cAMP receptor subtype 2, CAR2. CAR2 is structurally similar to CAR1. Overall, their transmembrane and loop domains are $\sim 75 \%$ identical in amino acid sequence; however, their carboxyl termini are quite dissimilar; CAR2 possesses homopolymeric runs of histidines and asparagines that are absent from the corresponding region in CAR1. Although CAR1 is maximally expressed during the early stages of development, CAR2 is expressed only after cells have aggregated and, then, preferentially in prestalk cells. Transgenic Dictyostelium that have had their wild-type CAR2 gene replaced by a defective copy using homologous recombination proceed through early development but are detained at the tight mound stage. CAR2 may be required for cAMP-directed sorting of prestalk cells during pattern formation within the aggregation mound. Furthermore, although prestalk genes are expressed normally in aggregates that lack CAR2, they exhibit an enhanced expression of prespore-specific mRNA. Previously, we had shown that there was a requirement for CAR1 during early development. The present results demonstrate that the multiple responses of Dictyostelium to cAMP are regulated by distinct cAMP receptors that are encoded by unique genes.

[Key Words: cAMP; receptors; gene expression; Dictyostelium]

Received August 14, 1992; revised version accepted November 30, 1992.

The development of Dictyostelium discoideum is initially characterized by a transition from a unicellular, vegetative organism to a multicellular aggregate with accompanying cytodifferentiation (for review, see Kimmel and Firtel 1991; Williams 1991). As cells stream into loose aggregation mounds, distinct prestalk and prespore cell types become evident; these cells are the progenitors to the terminally differentiated stalk and spore cells found in mature fruiting bodies at the culmination of development. During aggregate formation, a cellular pattern is also established as the prestalk cells sort to the dorsal and ventral regions of the aggregate. A tip of

\footnotetext{
${ }^{3}$ Present Address: Department of Anatomy and Cell Biology, Emory University School of Medicine, Atlanta, Georgia 30322 USA.

${ }^{4}$ Corresponding author.
}

prestalk cells then forms on the mound. This tip extends as the aggregate elongates into a migrating pseudoplasmodium, or slug, containing prestalk cells located primarily at the anterior or very posterior of the organism. Aggregation, differentiation, and pattern formation are regulated by response to extracellular cAMP. The extracellular cAMP serves as a signaling molecule to promote the accumulation of the intracellular messengers, cAMP, $\mathrm{IP}_{3}, \mathrm{Ca}^{++}$, and cGMP, by activating, respectively, adenylyl cyclase, phospholipase $\mathrm{C}, \mathrm{Ca}^{++}$transport, and guanylyl cyclase (Dinauer et al. 1980; EuropeFinner and Newell 1987; van Haastert et al. 1989; Milne and Coukell 1991). As a chemoattractant, cAMP mobilizes cells for adhesion and directs cell sorting within the aggregate (Konijn et al. 1967; Traynor et al. 1992). Extracellular cAMP is also required, along with other factors 
[e.g., differentiation inducing factor (DIF)], for developmentally regulated gene expression and cytodifferentiation (Schaap and van Driel 1985; Gomer et al. 1986; Haribabu and Dottin 1986; Kimmel and Carlisle 1986; Oyama and Blumberg 1986; Kimmel 1987; Williams et al 1987).

Appropriate cell density and depletion of amino acids from growth media trigger the initial phases of development and secretion of cAMP (see Gomer et al. 1991; Rathi et al. 1991). Cells begin to accumulate surface receptors that bind extracellular cAMP specifically and are linked through $\mathrm{G}$ proteins to the downstream effectors, including adenylyl cyclase (Schaap and Spek 1984; Theibert et al. 1986). Intracellular cAMP levels increase in response to receptor stimulation, and cAMP is secreted to relay the cAMP signal. With continued stimulation, receptors become desensitized and concomitantly hyperphosphorylated. Extracellular cAMP is degraded by membrane-bound and secreted phosphodiesterase (PDE), which attenuates the signal (see Franke et al. 1991). Receptor phosphorylation levels decrease, receptors regain sensitivity, and a new wave of cAMP signal-relay ensues (Klein et al. 1985, 1987; van Haastert 1987). These oscillating waves appear with periodicities of $\sim 6 \mathrm{~min}$. Providing shaking cultures of Dictyostelium with exogenous $20 \mathrm{nM}$ pulses of cAMP at 6 -min intervals to mimic normal oscillations promotes the early developmental events that are regulated by cAMP receptors. These include adenylyl cyclase activation and cGMP synthesis. If, however, cAMP is presented as a nonfluctuating stimulus during early development, these responses adapt and receptors down-regulate.

Secreted cAMP is also essential for cytodifferentiation and pattern formation (Schaap and van Driel 1985). Certain responses to cAMP in postaggregated cells may be fundamentally different than those during aggregation. Although many genes require pulses of cAMP for their appropriate developmental regulation early during development, genes expressed specifically in prespore cells may respond to a continuous, exogenous cAMP stimulus (Schaap and van Driel 1985; Gomer et al. 1986; Haribabu and Dottin 1986; Kimmel and Carlisle 1986; Oyama and Blumberg 1986; Kimmel 1987).

cAMP also directs the differential sorting of prestalk and prespore cells during aggregate formation (Traynor et al. 1992). Initially, both cell types appear scattered throughout the aggregation mound. Prestalk cells then begin a spiral migration toward the center of the mound surface in a pattern similar to that observed during aggregation (Esch and Firtel 1991; Siegert and Weijer 1991). This is consistent with prestalk cell movement to the presumptive tip in response to cAMP pulses (Traynor et al. 1992). The differences in movement of the prestalk and prespore cells are also pronounced within the migrating slug (Siegert and Weijer 1991, 1992). Prestalk cells continue to move in a spiral-like pattern within the slug anterior and perpedicular to the longitudinal axis. The periodic movement and shape change of these cells is, again, indicative of a role for cAMP in patterning. In contrast, the prespore cells move parallel to the anteriorposterior axis.
Studies using cAMP analogs have indicated that in most instances exogenous cAMP acts initially at surface receptors and not intracellularly (Schaap and van Driel 1985; Gomer et al. 1986; Haribabu and Dottin 1986; Oyama and Blumberg 1986; Kimmel 1987). Dictyostelium development thus appears to be regulated by multiple responses to cAMP acting through different receptor-mediated pathways. One of these pathways cycles through a responsive and desensitized state that is regulated by the low-level oscillations in extracellular cAMP that are observed during aggregation. Another pathway appears to be activated by continuous exposure to higher cAMP levels. It has not been clear whether these various responses result from the interaction of the same cAMP receptor form with multiple intracellular components or of distinct receptor subtypes. We had presented suggestive evidence in support of the latter (Saxe et al. 1991a). Here, we characterize a family of cAMP receptor genes related to $C A R 1$ and, in particular, describe the isolation of a gene, $C A R 2$, that encodes a cAMP receptor expressed after aggregation that plays an important role during the later stages of Dictyostelium development. These results demonstrate that a series of sequentially expressed cAMP receptors regulate the development of Dictyostelium.

\section{Results}

\section{The cAMP receptor gene family of Dictyostelium}

Previously, we have described the isolation of CAR1, a cAMP receptor gene from Dictyostelium that is expressed at high levels during the initial stages of development (Klein et al. 1988; Saxe, et al. 1991a). Under stringent conditions, CAR1 probes hybridize with a simple pattern to Dictyostelium genomic DNA blots indicative of a single-copy gene. When CAR 1 is hybridized at reduced stringency ( $25 \%$ formamide), a more complex pattern of bands is detected (see Fig. 1). From such data we suggested that multiple $C A R$ genes are present in the genome of $D$. discoideum. To examine this further, we screened genomic and cDNA libraries at low stringency using a CAR1 cDNA probe and isolated recombinants that hybridized relatively poorly to $C A R 1$. When probes $C A R 2$ and $C A R 3$ from different presumptive cAMP receptor sequences were hybridized at high stringency (50\% formamide) to genomic blots of Dictyostelium DNA, they detected a subset of the bands that had originally hybridized only weakly to CAR1 (see Fig. 1).

Figure 1 also shows that all of the bands that crosshybridize with CAR1 are not accounted for by CAR2 and $C A R 3$, suggesting the presence of an additional, related cAMP receptor gene. In a separate communication we discuss the characterization and function of the final member of this gene family, CAR4 (J. Louis, G. Ginsburg, and A. Kimmel, unpubl.). Although, these results limit the size of this family of receptors, they do not exclude the presence of more distantly related receptor species. 


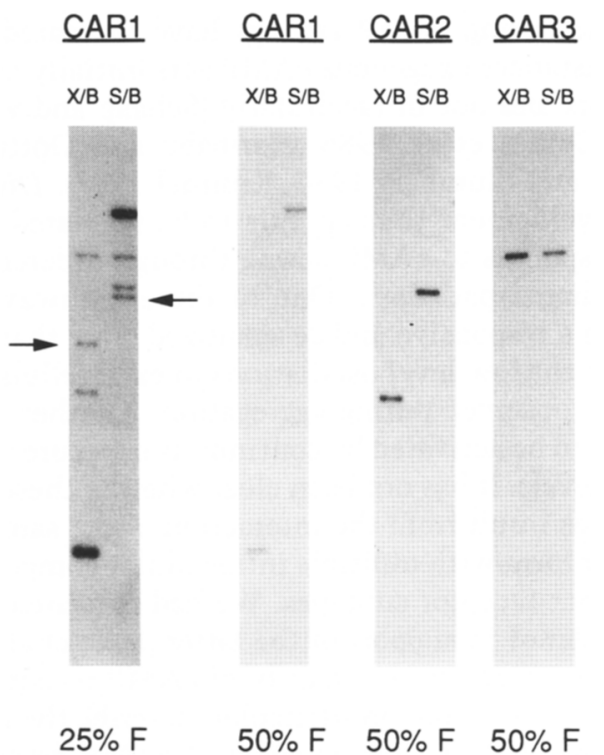

Figure 1. Hybridization of CAR Probes to $D$. discoideum genomic DNA. Genomic DNA was digested with $X b a \mathrm{I}$ plus $B s t X I$ (X/B) or ScaI plus BstXI (S/B) restriction enzymes, separated by gel electrophoresis, and blotted for hybridization with probes from CAR1, CAR2, or CAR3 DNAs. Hybridization reactions were incubated at $37^{\circ} \mathrm{C}$ in $0.8 \mathrm{M} \mathrm{Na}^{+}$with either $25 \%$ formamide $(25 \% \mathrm{~F})$ or $50 \%$ formamide $(50 \% \mathrm{~F})$, as indicated. Arrows indicate restriction fragments for the additional cAMP receptor gene CAR4.

\section{Sequence analysis of CAR2}

To determine the structure of CAR2 we sequenced both genomic and cDNA copies. Figure 2 shows the nucleic acid sequence of the entire protein-coding region and a portion of the $5^{\prime}$ - and $3^{\prime}$-untranslated regions. Except for a small (101-bp), A+T-rich intron located within the protein-coding region at the position equivalent to amino acid 100, the genomic and cDNA sequences are colinear. The deduced amino acid sequence of CAR2 indicates a protein of 375 amino acids. A most striking characteristic is the extended homopolymeric runs of histidines and asparagines located in the carboxy-terminal region. These derive from repeating runs of the codons CAT and AAT, respectively. We and others have noticed similar homopolymeric runs of repeating trinucleotide sequences in the coding as well as noncoding regions of several Dictyostelium genes (Kimmel and Firtel 1985; Shaw et al. 1989).

Although the cross-hybridization data strongly suggest that CAR2 is closely related to CAR1, they are, nonetheless, insufficient to confirm that CAR2 is a cAMP receptor. We have shown that vegetatively growing Dictyostelium that ectopically express CAR2 newly acquire several hundred thousand cAMP-binding sites on their cell surfaces (Johnson et al. 1992), and Johnson et al. (this issue) have demonstrated the photoaffinity labeling of expressed CAR2 protein by cAMP and its in vivo phosphorylation in cAMP-stimulated cells. Thus, CAR2 encodes a surface cAMP receptor.

\section{CAR2 is structurally similar to $G$ protein-coupled receptors}

The family of $\mathrm{G}$ protein-linked receptors are characterized by a common structural organization (see Klein et al. 1988). All of these receptors have seven regions that are enriched in hydrophobic amino acid residues. It is suggested that these regions form helices that span the lipid bilayer. We had shown previously that the deduced amino acid sequence of CAR1 included seven such regions that shared $20 \%$ amino acid identity with the seven putative transmembrane domains of bovine rhodopsin, another cell-surface receptor that couples to $\mathrm{G}$ proteins (Klein et al. 1988).

Figure 3 shows an amino acid alignment of CAR2 with CARl. The regions are compared according to the putative structural domains of CAR1. A 2-residue gap in the first extracellular loop is the only spacing difference required to align the two proteins. The regions with greatest amino acid identities are located within the trans-

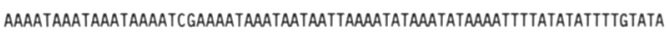

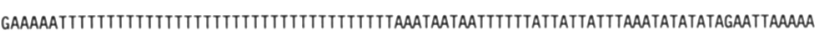
ATG ACT ATT ATG TCA GAT ATT ATC GCA CAA AGA ACA ATC TTG CTA ATC GCG GAT ITT TCA TCG ATI ATT GGA TGT TCT TTG GIT TTA AIT GGA ITT TGG AGG TTA AAA CIT TTA CGT AAT CAC ATT ACA AAA ATT ATA TCA TTA TUT TGC GCA ACT TCT CTA TTT AAA GAT GTA ATA TCA ACA ATT ATC ACA ITA CTG TAT AAA CCC GAC CAA ACA GAA TCT GGT IIT CCT TGI TAT CIT CAT GCA ATA GTA ATT ACT IIT GGT

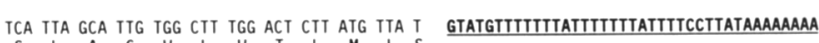
$S L_{A} C W_{L} W_{T} L_{M} L_{S}$

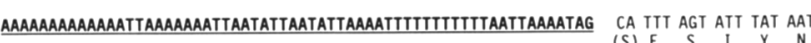
ITA ATA GIA AGA AGA GAA CCA GAA CCA GAA NGA TIT GAA AAA TIT TAT TTT TGT CTT TGT TAT GGA IIA CCA IIG ATT AGT ACG AIT GTT ATG TTA ICA ACA CAT AII AIT CAA CCA GIT GGT GGT TGG TGT ITA CCA ITG ATT AGT ACG AIT GTT ATG TTA TCA ACA CAT AIT AIT CAA CCA GIT GGT GGT TGG TGT

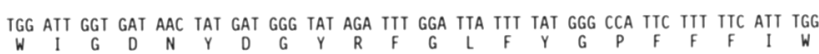
GGA ACA TCA GCA ATT ITG GIT GGA ITA ACA ICG AAA TAT ACA TAC TCT GTA ATA CGT AGT AGT GTA ICT GAT AAT AAG GAT AAA CAT ATG ACC TAT CAA TIT AAA TTG ATA AAT TAT ATC GTT GTA TTT ITG

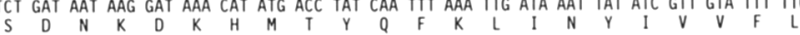
GIT TGT TGG GTA TIT GCA ATC GIT AAT CGT AIT CTA AAT GGT TTA AAT CAA TIT CCA ACG GIT CCA

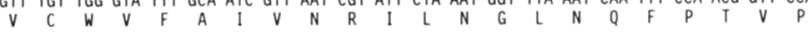

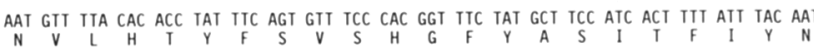
AAT CCA TTG ATG TGG CGT TAC IIT GGT GCA AAA ITC TTA ITG ATC ITT AGT AAA IIT GGT ITA TIT

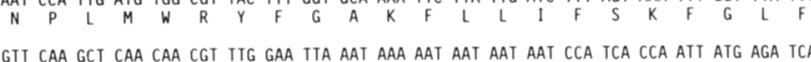
$\underset{V}{G I T}$ CAA GCT CAA CAA CGT TIG GAA TTA AAT AAA AAT AAT AAT AAT CCA TCA CCA ATI ATG AGA TCA AAG AAT GCT CTA GAT AAT GGT GCC GAC TCT TCG GIT GTA GAA TTA CCT TGT CTC TCA AAA GCT GAT TCT ITA TCA CTI GAT GCT GAA AAT AAT AIT GAA ACT CCA AAA GAA AAT GAA AAT CAA AAT CAT CAT

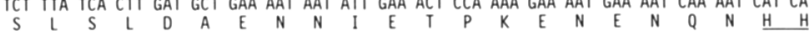
CAT CAT CAT CAT CAT CAT CAT CAT CAT AAT CAT TAT AAT AAT AAT AAC AAT AAT AAT AAT ATA AAT

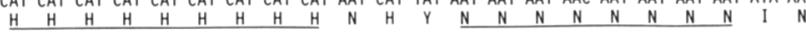
AAT AAA AAT GAT ATG AIT TAAAAGTGATAATAAAATAAATAATAAATAATCAATAATCAATAATAAGGITCAATATAITC N $\quad K \quad N \quad D \quad M$

ACACTAAACGGATTACTITItItigaAAAAAAAAAAAAAAAAAAAGAAAAAAAAAAAAAAAG

Figure 2. Nucleotide and derived amino acid sequences of $C A R 2$. The entire protein-coding region of CAR2 is indicated along with $5^{\prime}$ - and $3^{\prime}$-untranslated sequences. The homopolymeric runs of histidyl and asparginyl residues at the carboxyl terminus of the CAR2 protein are indicated by underline. The single intron of $101 \mathrm{bp}$ is in boldface type and underlined. 


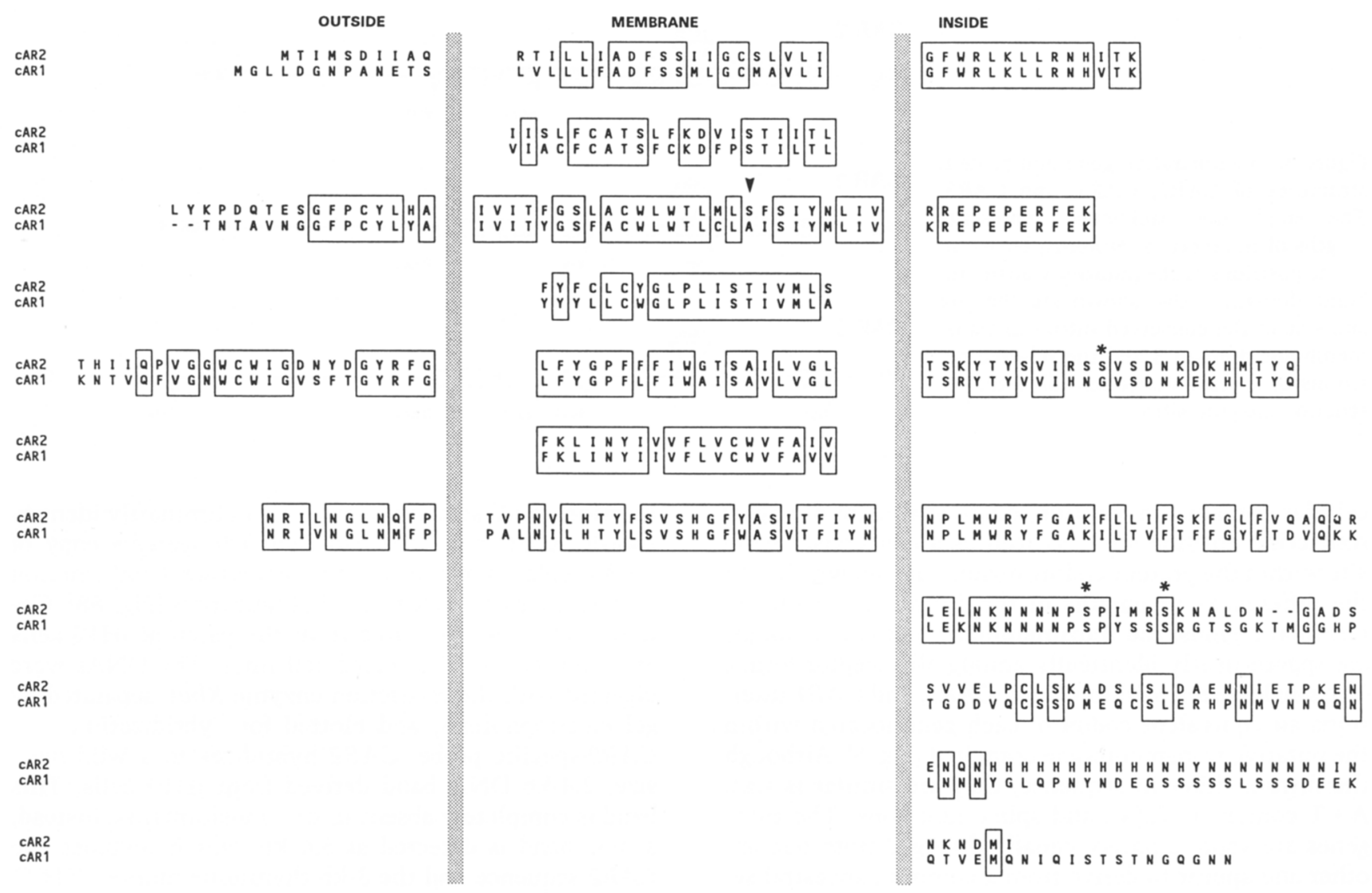

Figure 3. Amino acid sequence comparison between CAR2 and CAR1. Amino acid identities are enclosed in boxes. Sequences are displayed with extracellular (outside) loops, intracellular (inside) loops, and transmembrane domains according to the topological structure predicted for CAR1 (Klein et al. 1988). The splice junction conserved in both genes is indicated by the downward arrowhead; an asterisk $\left({ }^{*}\right)$ indicates potential seryl phosphorylation sites for CAR2.

membrane and cytoplasmic loop domains. Overall, these regions are $>70 \%$ identical in amino acid sequence, although certain regions are clearly more related than others. When conservative amino acid comparisons are also considered, CAR 1 and CAR 2 are $\sim 85 \%$ similar in the transmembrane and loop domains. Although considerable sequence identity extends partially into the carboxyl terminus, the $\sim 70$ amino acids at the carboxyl terminus and the entire amino terminus of CAR2 do not share sequence identity with CAR1. The homopolymeric runs of histidines and asparagines observed in the carboxyl terminus of CAR2 are absent from CAR1. Although seryl residues are distributed throughout the third cytoplasmic and carboxy-terminal domains of CAR2, the clusters of seryl residues characteristic of CARl are not observed. Two potential phosphorylation sites in this region are shared by CAR1 and CAR2 (see Fig. 3). The single potential target (Arg-X-Ser) for protein kinase A phosphorylation located in the third cytoplasmic loop of CAR2 is absent in the corresponding region of CAR1 but is similarly localized in CAR3 (see Fig. 3).

Because the transmembrane domain regions of CAR2 and CAR1 were not entirely identical, it was necessary to show that the CAR2 domains were nevertheless suf- ficiently enriched in hydrophobic residues to theoretically span a lipid bilayer. When analyzed in conjunction with CAR1, the hydropathicity plots are nearly identical. Predominant hydrophilic regions of each protein represent cytoplasmic domains.

\section{Structural similarities among the cAMP receptor gene family}

In Johnson et al. (this issue), we describe the sequence and structural analysis of CAR3. CAR2, CAR1, and CAR3 comprise distinct subtypes of the $G$ proteinlinked cAMP receptor family of $D$. discoideum and are predicted to form substantially identical structures. Their close similarity is evident, although certain transmembrane and loop domains are more conserved than others. Conceivably the conserved or diverged regions are involved, respectively, in their shared or distinct functions.

Additional relationships among the CAR family members are seen with comparisons among their overall genomic organizations. Figure 4 combines genomic and protein structural information. Boxes indicate transmembrane domains, and lines indicate intra- and extra- 


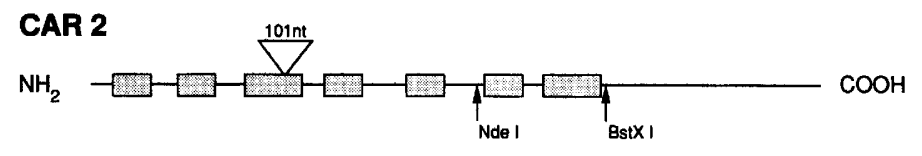

Figure 4. Comparative gene and protein structures of CAR2, CAR1, and CAR3. The single-line backbones represents lengths of the receptor proteins; boxes indicate positions of the putative transmembrane domains. Also shown are the site and size of the conserved intron in transmembrane domain 3 and the relative positions of several shared and diverged restriction enzyme sites.
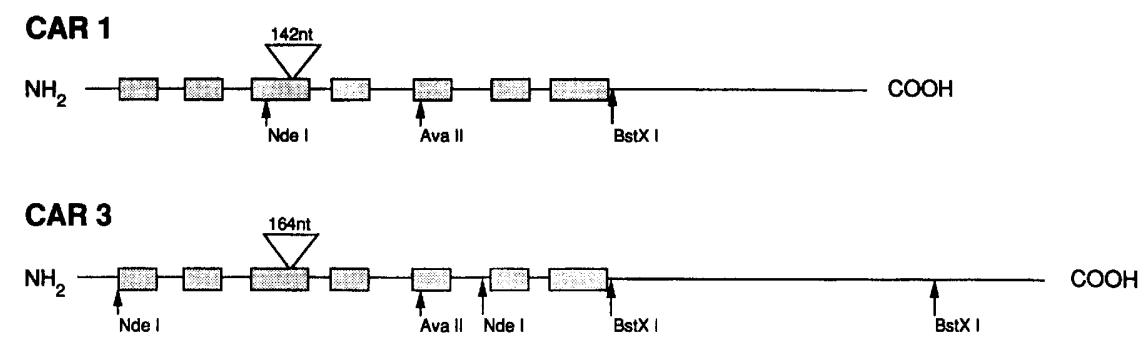

cellular domains. In addition, major shared (or diverged) restriction enzyme sites, as well as a conserved intron site within the protein-coding regions, are shown. But for the differences in lengths of the amino- and carboxyterminal regions, the transmembrane and loop domains are spaced nearly identically among all receptor forms. The single intron within CAR2, CAR3, and CAR1 interrupts an equivalent codon of each gene located within the putative transmembrane domain 3 (Fig. 5). Although the introns differ in sequence, they are similar is size, $A+T$ content $(\sim 95 \%)$, and splice junctions. The three genes are approximately equally diverged from one another and appear to derive from a common ancestral sequence.

\section{Gene disruption of CAR2}

The Dictyostelium marker THY1 (Dynes and Firtel 1989|, which permits growth of cells in the absence of thymidine, was inserted into a genomic fragment of $C A R 2$. The site of integration interrupted an amino acid sequence identically conserved in CAR $1-4$ (see Fig. 6A). Such disrupted cAMP receptor genes cannot produce a functional receptor protein (Sun and Devreotes 1991). The Dictyostelium strain JH10 (Hadwiger and Firtel 1992) that is auxotrophic for thymidine was transformed with the linearized CAR2 fragment carrying the THY1 marker; cells were selected that were able to grow in the absence of exogenous thymidine. A polymerase chain

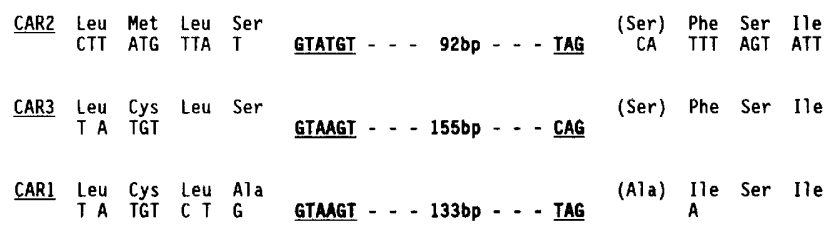

Figure 5. Splice junction identitites among $C A R 2, C A R 3$, and $C A R 1$. Nucleotide and amino acid sequence comparisons surrounding the intron that interrupts the region encoding transmembrane domain 3 . The nucleotide sequence is listed for $C A R 2$; only those nucleotides that differ in CAR3 or CAR1 are indicated. The intron junction and remaining nucleotide lengths are in boldface type and underlined. reaction (PCR) screen was used to preliminarily identify transformants that had had their endogenous copy of $C A R 2$ replaced by the disrupted construct. Confirmation was achieved by DNA blot hybridization (Fig. 6B). Genomic DNA was isolated from the parental JH10 cells and from two PCR-selected cell lines. The DNAs were digested with the restriction enzyme $X b a I$, separated by gel electrophoresis, and blotted for hybridization to a CAR2-specific probe. CAR2 hybridizes to a wild-typesize, 2.4-kb DNA band derived from JH10 cells. This band is completely absent in the transformants. Instead, a new band is detected at $5.4 \mathrm{~kb}$, which includes the CAR2 sequence and the $3-\mathrm{kb}$ thymidine marker; THY1 does not contain any $\mathrm{XbaI}$ sites. Thus, the transformants car2-ko1 and car2-ko6 have had their endogenous CAR2 gene substituted by a disrupted copy. The results indicate that no additional copies of the disrupted CAR2 gene were inserted into the genome and that the phenotypes of car2-ko mutants are solely the result of CAR2 disruption.

Previous data based on RNA blot hybridization, PCR amplification, and library screening suggest that the initial accumulation of CAR2 mRNA takes place at the approximate time of tip formation (Saxe et al. 1991b). RNAs from the less dense, prestalk cell fraction of slugs are highly enriched $(\sim 10$-fold) in CAR2 mRNA relative to RNAs from the denser, prespore cells (Saxe et al. 1991b). Furthermore, cytological staining for $\beta$-galactosidase activity using CAR2 promoter-lacZ fusions indicates CAR2 expression in prestalk cells (C. Saxe, unpubl.).

We examined the expression pattern of $C A R 2$ during development of parental and CAR2 disrupted cell lines (Fig. 7). Poly(A) ${ }^{+}$mRNA was isolated from Dictyostelium at major morphological stages, separated by gel electrophoresis under denaturing conditions, and hybridized at high stringency to a $C A R 2$ probe. No $C A R 2$ mRNA is detected in growing cells $(0 \mathrm{hr})$, in aggregating cells $(5 \mathrm{hr})$, or during early formation of multicellular aggregates $(10$ $\mathrm{hr})$. After tip formation ( $15 \mathrm{hr})$, a single $2-\mathrm{kb}$ CAR2 mRNA is expressed in the parental JH10. Similar levels of expression persist through the early stages of culmination $(20 \mathrm{hr})$. Similar analyses of expression of CAR2 were performed using car2-ko1 and car2-ko6 cell lines. 


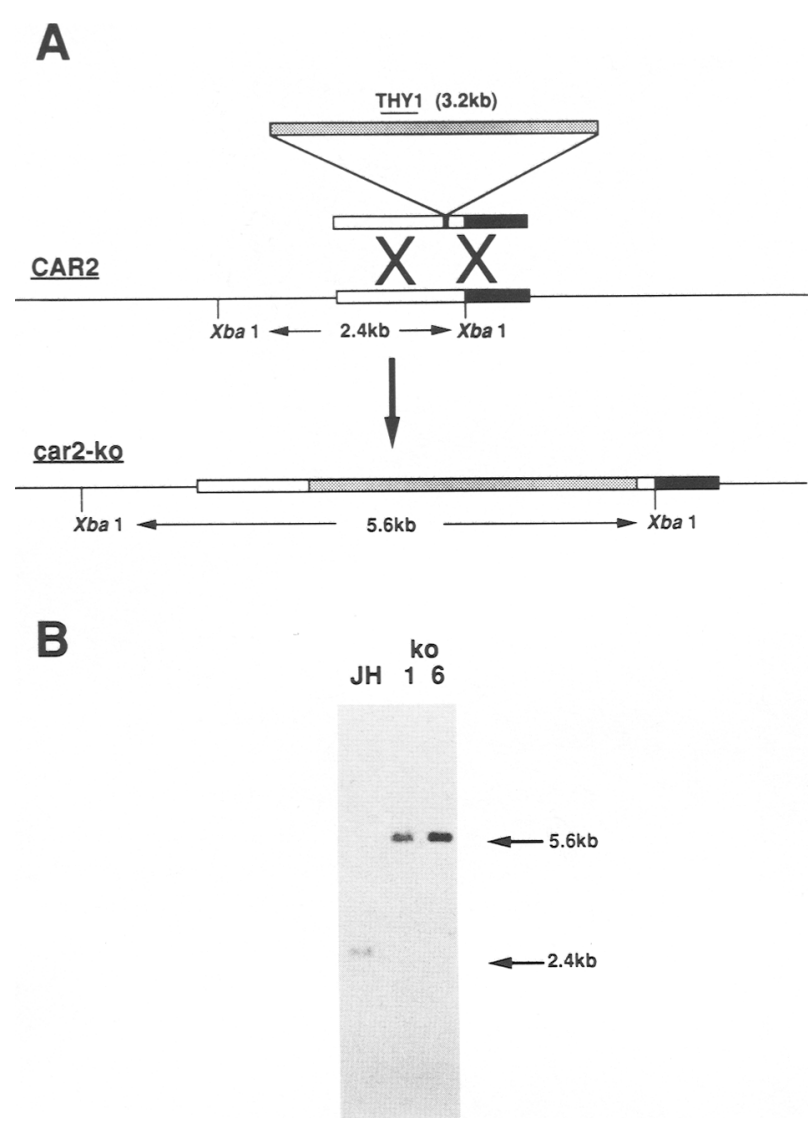

Figure 6. Procedure for generating the CAR2 disruption by homologous recombination. $(A)$ Diagram of the CAR2 gene with sequences located upstream and downstream of the single $\mathrm{XbaI}$ site within $C A R 2$ indicated by open bars and solid bars, respectively. Transformation vector containing the THY1 gene within $C A R 2$ is indicated as are the regions predicted to undergo a double crossover. The resulting disrupted car2-ko containing the THY1 marker is also shown. (B) Blot hybridization of DNA from $\mathrm{JH} 10$ parental and car2-ko1 and car2-ko6. DNAs were digested with $X b a I$ and hybridized with an upstream CAR2 probe (see Fig. 8A). The endogenous $X b a \mathrm{I}$ band of JH10 migrates at $2.4 \mathrm{~kb}$; the disrupted car2-ko band is $5.6 \mathrm{~kb}$.

Cells were developed in parallel with parental JH10 cells and RNAs isolated at identical times; however (see below), morphological structures were not equivalent. In neither car2 ${ }^{-}$cell line could we detect the expression of a CAR2 mRNA species. Thus, the cell lines disrupted for CAR2 are completely defective in CAR2 production.

\section{Dictyostelium cells with a defective CAR2 develop abnormally and cannot form tips}

$\mathrm{JH} 10$ cells and car2- ${ }^{-}$cells were developed on agar plates, and their morphologies were monitored. car2-ko1 development precisely parallels control JH10 through the first $11 \mathrm{hr}$ (Fig. 8A,B). Cells stream and form tight aggregation mounds with appropriate kinetics. Tip structures of $\mathrm{JH} 10$ are first observed at $\sim 11 \mathrm{hr}$, and migrating slugs appear by $16 \mathrm{hr}$ (see Fig. $8 \mathrm{C}, \mathrm{D}$ ). Greater than $90 \%$ of the original JH10 aggregates are able to complete development and form mature fruiting bodies by $29 \mathrm{hr}$. car2 ${ }^{-}$ cell lines exhibit abnormal patterns, with development arresting after tight aggregates form. Tips that are normally seen after $12 \mathrm{hr}$ do not appear in car2- cells (Fig. $8 \mathrm{E})$. After an extended period of time many car2 ${ }^{-}$aggregates extend a finger-like projection, and eventually $(>150 \mathrm{hr}$ ) produce a number of deformed fruiting bodies. Thus, the prestalk-specific, cAMP receptor CAR2 plays a key role during tip formation and late development, although it is possible to proceed through this stage, albeit abnormally, in its absence.

\section{Aggregates of car2- cells overexpress prespore but not prestalk $m R N A$}

We examined the expression of cell-type specific genes in developed $\mathrm{car}^{-}$cells to determine whether the inability to form tip structures resulted from an inability to undergo cytodifferentiation. We selected markers with distinct spatial and temporal patterns of expression. The prestalk markers $e \mathrm{~cm} A$ and $e \mathrm{~cm} B$ are induced by DIF and initially appear in aggregated cells (Williams et al. 1987). The ecmA gene is primarily expressed in the prestalk A cells that migrate apically during tip formation (see Williams 1991). The prestalk B cells sort to the base of the aggregation mound. During culmination, the levels of $e \mathrm{cmB}$ mRNA increase as prestalk A cells enter the stalk tube and begin to express ecmB. The $2 \mathrm{H}-6$ and the prespore 14E- 6 genes are induced by cAMP in shaking cultures but are maximally expressed at different developmental times (Mehdy et al. 1983; Ginsburg and Kimmel 1989|.

Figure 9A shows developmental expression patterns for ecmA, ecmB, 2H-6, and 14E-6 mRNAs in the parental $\mathrm{JH} 10$ and car2-ko1 cell lines. The results for $\mathrm{JH} 10$ are typical for these genes. The prestalk markers $e \mathrm{~cm} A$ and $e c m B$ apppear to induce normally in car2-ko1 cells. This would suggest that the prestalk A and B cells differentiate in the car2 $2^{-}$cells $\mathrm{t}$ o not sort properly. Although $e \mathrm{~cm} A$ reaches equivalent levels of expression in $\mathrm{JH1O}$ and car2-ko1 cells, ecmB is only expressed at low levels

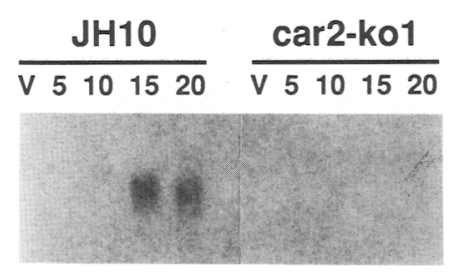

Figure 7. Developmental expression of CAR2 mRNA in control and car2- Cells. JH10 and car2-ko1 cells were harvested in logarithmic phase (V), washed, plated on an agar surface, and allowed to proceed synchronously through development. At 5-hr intervals, cells were collected and RNA was isolated. The RNAs were size separated on denaturing gels and blotted for hybridization to CAR2. 
A

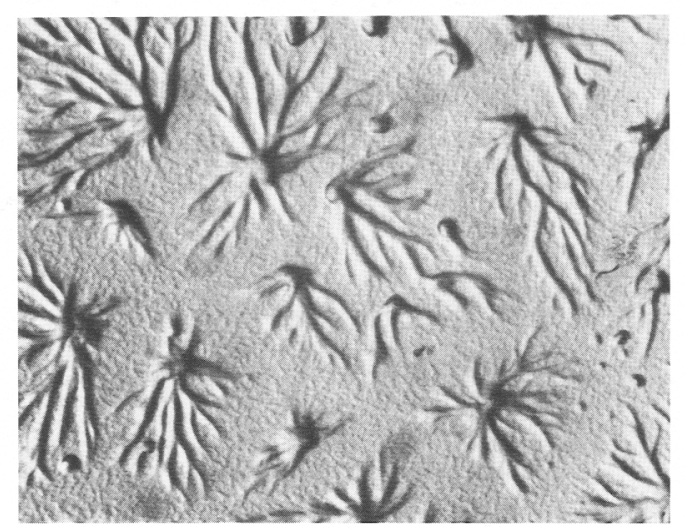

B

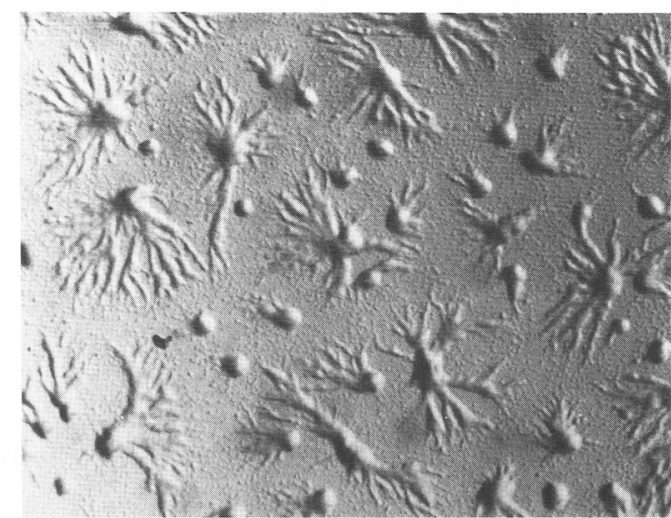

C

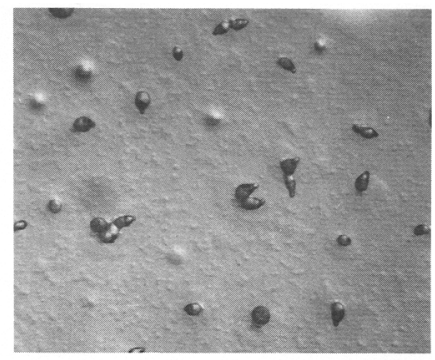

D

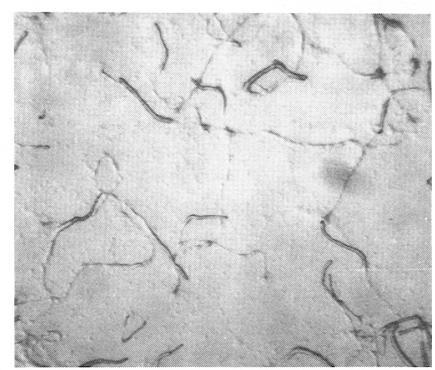

E

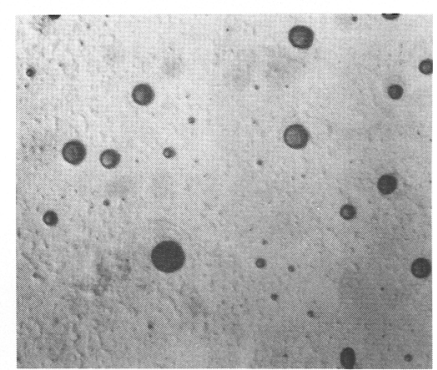

Figure 8. Developmental morphologies of control and car2 ${ }^{-}$cells. JH10 and car2-ko1 cells were harvested in logarithmic phase, washed, plated on filter pads, and allowed to proceed synchronously through development. $(A) \mathrm{JH} 10$ cells at $9 \mathrm{hr}$ of development; $(B)$ car2-ko1 cells at $9 \mathrm{hr}$ of development; $(C) \mathrm{JH} 10$ cells at $12 \mathrm{hr}$ of development; $(D) \mathrm{JH} 10$ cells at $19 \mathrm{hr}$ of development; $(E)$ car2-ko1 cells at $19 \mathrm{hr}$ of development.

in car2 $2^{-}$lines. Because the car2 ${ }^{-}$lines do not culminate, it seems likely that high levels of expression are not acheived because of the inability to further increase $e \mathrm{~cm} B$ expression during stalk tube formation.

$2 \mathrm{H}-6$ expression is regulated comparably in both cell lines. The slightly diminished expression observed in car2-ko1 is probably not significant. Conversely, a dramatic difference in expression level is observed for the prespore gene 14E-6. Here, 14E-6 mRNA accumulates to levels that are $\sim 10$-fold greater in car2-ko1 cells than in parental cells, although its temporal regulation is similar in both strains.

To determine whether the regulation of $14 \mathrm{E}-6$ was similarly affected in shaking culture, we differentiated car2-ko1 cells in the presence of a saturating concentration $(300 \mu \mathrm{M})$ of exogenous cAMP. Again, we observe a significant overexpression of $14 \mathrm{E}-6$ in car2 ${ }^{-}$cells compared with $\mathrm{JH} 10$. No differences in expression were seen for the cAMP-regulated genes $2 \mathrm{H}-6$ or $10 \mathrm{C}-3$ that are not prespore specific.

\section{Discussion}

Aggregation and differentiation of Dictyostelium are in part regulated by extracellular cAMP acting as a chemoattractant and first messenger (see Kimmel and Firtel 1991). Many of the effects of extracellular cAMP are mediated through cell-surface receptors linked to G proteins. On the basis of low-stringency hybridization of Dictyostelium genomic DNA with a probe for cAMP receptor CAR1, we had suggested previously the presence of a family of cAMP receptor genes, but it had been unresolved whether the multiple roles of extracellular cAMP were regulated by different receptor subtypes with separate functions (Saxe et al. 1991a). In this paper and in Johnson et al. (this volume) we describe the isolation of $C A R 2$ and $C A R 3$, two new members of that gene family and assign to them distinct roles for cAMP regulation during the development of $D$. discoideum.

Ectopic expression of CAR1, CAR2, and CAR3 has established them as bona fide cAMP cell-surface receptors, and hydropathy analysis suggests that they similarly span the membrane seven times and couple to $G$ proteins (Johnson et al. 1992). A comparison of the deduced amino acid sequences of these receptors shows $\sim 75 \%$ identity through the transmembrane and loop domains and partially into the cytoplasmic carboxyl termini. The carboxy- and amino-terminal regions, however, are 

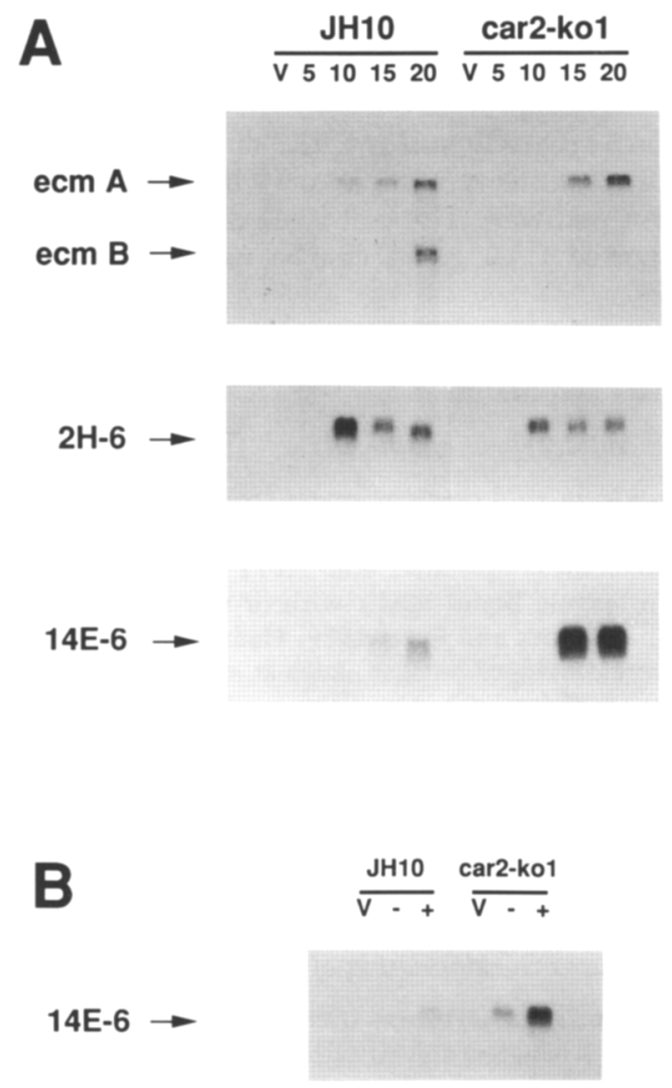

Figure 9. Expression of developmentally regulated genes in control and car2- cells. $(A) \mathrm{JH10}$ and car2-ko1 cells were harvested in logarithmic phase (V), washed, plated on an agar surface, and allowed to proceed synchronously through development. At 5-hr intervals, cells were collected and RNA was isolated. The RNAs were size separated on denaturing gels and blotted for hybridization to probes for the prestalk genes ecm $A$ and $e c m B$, the prespore gene 14E-6, and the cAMP-regulated gene $2 \mathrm{H}-6 .(B) \mathrm{JH} 10$ and car2-ko1 cells were harvested in logarithmic phase $(\mathrm{V})$, washed, and differentiated in liquid culture for $10 \mathrm{hr}$ in the absence $|-|$ or presence $|+|$ of cAMP. The RNAs were size separated on denaturing gels and blotted for hybridization to a probe for the prespore gene $14 \mathrm{E}-6$.

largely distinct both in overall length and sequence. The extremely close similarity in gene organization among these receptors suggests a relatively recent divergence from a common ancestor.

CAR2, CAR3, and CAR1 can undergo ligand-induced phosphorylation. The carboxy-terminal region of CAR1 contains clusters of seryl residues that have been implicated in ligand-induced phosphorylation of CAR1 (Klein et al. 1988). Of these, only serines at positions 298 and 303 are also present in CAR2 (see Fig. 3). It is noteworthy that these two serines are conserved as well in CAR3 and in CAR4, although any ascribed function is speculative (Klein et al. 1988; Johnson et al. 1992; J. Louis, G.T. Ginsburg, and A.R. Kimmel, unpubl.). A weak consensus (Arg-X-Ser) sequence for the cAMP-dependent protein kinase is also present in the third cytoplasmic loop of
CAR2, CAR3, and CAR4 but not CAR1 (Klein et al. 1988; Johnson et al. 1992; J. Louis, G.T. Ginsburg, and A.R. Kimmel, unpubl.). Similarly, CAR2, CAR3, and CAR4 contain homopolymeric amino acid clusters in their carboxy-terminal region (Klein et al. 1988; Johnson et al. 1992; J. Louis, G.T. Ginsburg, and A.R. Kimmel, unpubl.). Such homopolymers are seen in a number of other Dictyostelium and non-Dictyostelium proteins (Kimmel and Firtel 1985). The significance, if any, of these is unknown.

$C A R 2$ and CAR1 have somewhat complementary patterns of expression. CAR 1 is maximally expressed at 5-10 hr of development. CAR1 mRNA levels then decline dramatically, although CAR1 mRNA is still detected at culmination. In contrast, CAR2 is only expressed in postaggregation stages. Nonetheless, the levels of CAR2 and CAR1 mRNAs are similar at these late stages of development after cytodifferentiation and pattern formation have occurred. CAR1 mRNA is expressed throughout development, whereas $C A R 2$ is a prestalk marker.

The expression patterns of CAR1 and CAR2 would suggest different developmental roles for each. CAR1 is maximally expressed during aggregation, when chemotaxis and cAMP relay and signaling are maximal (Saxe et al. 1991a). Consistent with this pattern of expression, disturbance of CAR1 expression by either antisense RNA or gene disruption causes development to arrest before aggregation (Klein et al 1988; Sun and Devreotes 1991). CAR2 RNA, on the other hand, is expressed only after aggregation is completed and loss of CAR2 expression has no effect on the ability of the organism to aggregate; but starting with tip formation, postaggregative development and morphogenesis are strongly affected.

A surprising phenotype of car2- cells is the capacity to overexpress the prespore-specific gene 14E-6. Because only $\sim 20 \%$ of differentiated cells are normally prestalk, this phenotype cannot be explained simply by an alteration in cell proportioning and a recruitment of prestalk cells into a prespore pathway. Furthermore, prestalk genes appear to be expressed similarly in both parental and car $2^{-}$cell lines. Although data would indicate otherwise, it is possible that only a small percentage of prespore cells express 14E-6 mRNA. This population may be larger in car2 $2^{-}$cell lines. It would also seem that this phenotype cannot be attributed to a difference in extracellular cAMP metabolism because overexpression of $14 \mathrm{E}-6$ is observed in shaking cultures treated with saturating concentrations of exogenous cAMP. Localization studies of $C A R 2$ gene expression would suggest that the modulation of prespore gene expression by CAR 2 must be mediated through prestalk cells. Overexpression of prespore genes may result indirectly from aberrant positioning of prestalk cells within the aggregation mound, although such an effect must be mimicked in agglomerates that form during differentiation in shaking cultures. DIF will inhibit prespore expression, and it is possible that DIF levels are affected in car2 ${ }^{-}$cells (Berks and Kay 1990). However, such an effect cannot be sufficient to diminish the positive role DIF plays in the induction of 
ecmA. Alternatively, an extremely low-level expression of CAR2 in prespore cells may directly regulate prespore gene expression.

It is also clear that CAR2 is not essential for the induction of expression of the cAMP-regulated genes $2 \mathrm{H}-6$, 14E-6, and 10C-3 by exogenous CAMP (Mehdy et al 1983; Ginsburg and Kimmel 1989|. Data suggest that such cAMP regulation is mediated by cell-surface cAMP receptors (Schaap and van Driel 1985; Gomer et al. 1986; Haribabu and Dottin 1986; Kimmel and Carlisle 1986; Oyama and Blumberg 1986; Kimmel 1987). Because car3 $^{-}$cells are capable of completing development (Johnson et al., this issue) it appears that receptors other than CAR2 and CAR3 are required for the observed regulation of gene expression by extracellular cAMP.

The differences in phenotypes between CAR1 and CAR2 gene disruptions underscore additional points about cAMP effects on development. Mutation of either gene causes an impairment of development. The receptors are clearly mediating significant and separate decisions for Dictyostelium development. The binding of cAMP to both of these receptors, therefore, is critical for proper developmental progress. Because CAR1 and CAR3 are expressed at similar, albeit low, levels in control and car2 ${ }^{-}$cells, they must not be interchangeable with CAR2.

The level of extracellular cAMP varies dramatically during aggregation. In the first few hours extracellular concentrations remain relatively low. However, by the time tight mounds form, cAMP levels have risen $>10$ fold (Abe and Yanagasawa 1983). At that stage, maximal signaling is restricted to the upper surface of the aggregate, a site to which the prestalk cells are primarily chemotactic (Siegert and Weijer 1991, 1992). In parallel with the increase in extracellular cAMP, cells alter their pattern of receptor sensitivity to cAMP. The relative affinity of CARl for cAMP is $>10$ times higher than is observed for CAR2 (Johnson et al. 1992). Consistent with these observations it is possible that the low-affinity, prestalk-specific receptor CAR2 is involved in cAMPdirected patterning of prestalk cells as they sort before and during tip formation. Conversely, cAMP levels at this time may be saturating for both CAR1 and CAR3; thus, they would be unable to monitor a cAMP gradient necessary for directed movement. It is interesting that aggregates that overexpress PDE have slightly reduced levels of cAMP and are unable to form normal tipped structures (Traynor et al. 1992). Yet, these prestalk cells retain their ability to migrate toward an ectopic cAMP source.

The three cAMP receptors may interact with different intracellular components and/or activate different signaling pathways. Alternatively, CAR1, CAR2, and CAR3 may activate similar pathways but only at restricted sensitivities to extracellular cAMP. The close structural and sequence similarities among the three receptor forms suggest common interactions with $G$ proteins. Recently, genes for the $\alpha$ subunits of $G$ proteins have been isolated from Dictyostelium (Pupillo et al. 1989; Hadwiger et al. 1991; Wu and Devreotes 1991).
Some of their expression patterns specifically overlap that of CAR2. It will be of interest to establish whether any of these $G$ protein components interact specifically with CAR2, CAR1, and/or CAR3 during postaggregative Dictyostelium development or whether they share a common pathway. The cell lines disrupted for each of the reported CAR genes can be used to address such questions.

\section{Materials and methods \\ Growth and development of cells}

$D$. discoideum wild-type strain was grown on bacteria and developed on filters as described previously (Ginsburg and Kimmel 1989). thy $1^{-}$parental and THY1 selected strains were grown in axenic liquid media with or without $100 \mu \mathrm{g} / \mathrm{ml}$ of thymidine (Dynes and Firtel 1989; Hadwiger and Firtel 1992). Cells were developed on agar plates at a surface density of $10^{7}$ cells $/ \mathrm{cm}^{2}$ or differentiated in shaking culture at a density of $10^{7}$ cells $/ \mathrm{ml}$ in $10 \mathrm{~mm}$ sodium phosphate (pH 6.4), $2 \mathrm{mM} \mathrm{MgCl}_{2}, 0.2$ $\mathrm{mM} \mathrm{CaCl}_{2}$. For cAMP treatment, shaking cultures received 20 $\mathrm{nM}$ pulses of cAMP at 6-min intervals for $3 \mathrm{hr}$; subsequently, the cultures were adjusted to and maintained at $300 \mu \mathrm{M}$ cAMP for the remainder of differentiation (Kimmel 1987).

\section{DNA-mediated transformation of Dictyostelium}

The thy $1^{-}$cell line JH10 was transformed to THY1 essentially as described (Hadwiger and Firtel 1992). Greater than $50 \%$ of the transformants isolated exhibited homologous recombination within the $C A R 2$ locus.

\section{Isolation and hybridization of RNA and DNA}

Total and poly $(\mathrm{A})^{+}$RNA were prepared from cells as described previously (Kimmel 1987). RNA was size separated on formaldehydeagarose gels and transferred to nitrocellulose (Kimmel 1987). Genomic DNA was isolated as described (Kimmel and Firtel 1985). DNA was size separated on agarose gels, transferred to nitrocellulose, and hybridized to radiolabeled probes. Probes were radiolabeled by the random primer method using $\left[\alpha^{32} \mathrm{P}\right]-\mathrm{dCTP}$ and hybridized to DNA blots, RNA blots, genomic libraries, and cDNA libraries. Hybridizations were performed at $37^{\circ} \mathrm{C}$ in $0.8 \mathrm{M} \mathrm{Na}^{+}$. High stringency buffers additionally contained $50 \%$ formamide, and reduced stringency buffers contained $25 \%$ formamide.

\section{DNA sequencing}

Sequencing was done by the dideoxy chain-termination method (Saxe at al. 1991a) as applied to double-stranded DNA.

\section{Acknowledgments}

We thank R. Firtel and J. Hadwiger for assistance with thymidine selection and the laboratories of R. Firtel and J. Williams for providing probes for developmentally regulated RNAs. We also appreciate the helpful comments and discussion of R. Gollop, R. Kay, C. Londos, D. Richardson, T. Sargent, and R. Simpson.

The publication costs of this article were defrayed in part by payment of page charges. This article must therefore be hereby marked "advertisement" in accordance with 18 USC section 1734 solely to indicate this fact. 


\section{Note added in proof}

The nucleotide and amino acid sequence data for CAR2 have been submitted to the EMBL/GenBank data libraries.

\section{References}

Abe, K. and K. Yanagasawa 1983. A new class of rapidly developing mutants in Dictyostelium discoideum: Implications for cAMP metabolism and cell differentiation. Dev. Biol. 95: $200-210$.

Berks, M. and R. Kay. 1990. Combinatorial control of cell-differentaition by cAMP and DIF-1 during development of Dictyostelium discoideum. Development 110: 977-984.

Dinauer, M.C., T.L. Steck, and P.N. Devreotes. 1980. Cyclic 3',5'-AMP relay in Dictyostelium discoideum. Adaptation of the cAMP signaling response during cAMP stimulation. $J$. Biol. Chem. 86: 554-561.

Dynes, J.L. and R.A. Firtel. 1989. Molecular complementation of a genetic marker in Dictyostelium using a genomic DNA library. Proc. Natl. Acad. Sci. 86: 4786-4790.

Esch, R.K. and R.A. Firtel. 1991. cAMP and cell sorting control the spatial expression of a developmentally essential celltype-specific ras gene in Dictyostelium. Genes \& Dev. 5: 921.

Europe-Finner, G.N. and P.C. Newell 1987. cAMP stimulates accumulation of inositol trisphosphate in Dictyostelium. I. Cell. Sci. 87: 221-229.

Franke, J., M. Faure, L. Wu, A.L. Hall, G.J. Podgorski, and R.H. Kessin 1991. Cyclic nucleotide phosphodiesterase of Dictyostelium discoideum and its glycoprotein inhibitor: Structure and expression of their genes. Dev. Genet. 12: 104-112.

Ginsburg, G. and A.R. Kimmel. 1989. Inositol trisphosphate and diacylglycerol can differentially modulate gene expression in Dictyostelium. Proc. Natl. Acad. Sci. 86: 9332-9336.

Gomer, R.H., D. Armstrong, B.H. Leichtling, and R.A. Firtel. 1986. cAMP induction of prespore and prestalk gene expression in Dictyostelium is mediated by the cell-surface cAMP receptor. Proc. Natl. Acad. Sci. 83: 8624-8628.

Gomer, R.H. I.S. Yuen, and R.A. Firtel. 1991. A secreted $80 \times 10^{3} \mathrm{M}_{\mathrm{r}}$ protein mediates sensing of cell density and the onset of development in Dictyostelium. Development 112: 269-278.

Hadwiger, J.A. and R.A. Firtel. 1992. Analysis of $\mathrm{G} \alpha 4$, a G protein subunit required for multicellular development in Dictyostelium. Genes \& Dev. 6: 38-49.

Hadwiger, J.A., T.M. Wilkie, M. Stratmann, and R.A. Firtel. 1991. Identification of Dictyostelium G $\alpha$ genes expressed during multicellular development. Proc. Natl. Acad. Sci. 88: 8213-8217.

Haribabu, B. and R.P. Dottin. 1986. Pharmacological characterization of cAMP receptors mediating gene regulation in Dictyostelium discoideum. Mol. Cell. Biol. 6: 2402-2408.

Johnson, R.L., P.J.M. van Haastert, A.R. Kimmel, C.L. Saxe, B. Jasdorff, and P.N. Devreotes. 1992. The cyclic nucleotide specificity of three cAMP receptors in Dictyostelium. I. Biol. Chem. 267: 4600-4607.

Kimmel, A.R. 1987. Different molecular mechanisms for cAMP regulation of gene expression during Dictyostelium development. Dev. Biol. 122: 163-171.

Kimmel, A.R. and B. Carlisle. 1986. A gene expressed in undifferentiated vegetative Dictyostelium is repressed by developmental pulses of cAMP and reinduced during dedifferentiation. Proc. Natl. Acad. Sci. 83: 2506-2510.

Kimmel, A.R. and R.A. Firtel. 1985. Sequence organization and developmental expression of an interspersed, repetitive ele- ment and associated single-copy DNA sequences in Dictyostelium discoideum. Mol. Cell. Biol. 5: 2123-2130.

- 1991. cAMP signal transduction pathways regulating development of Dictyostelium discoideum. Curr. Opin. Genet. Dev. 1: 383-390.

Klein, P.S., A., Theibert, D. Fontana, and P.N. Devreotes. 1985. Identification and cyclic-AMP induced modification of the cAMP receptor in Dictyostelium discoideum. I. Biol. Chem. 260: $1757-1764$.

Klein, P.S., R. Vaughan, J. Borleis, and P.N. Devreotes. 1987. The surface cAMP receptor in Dictyostelium. Levels of ligand-induced phosphorylation, solubilization, identification of primary transcript, and developmental regulation of expression. J. Biol. Chem. 262: 358-364.

Klein, P.S., T.J. Sun, C.L. Saxe, A.R. Kimmel, R.L. Johnson, and P.N. Devreotes. 1988. A chemoattractant receptor controls development in Dictyostelium discoideum. Science 241: 1467-1472.

Konijn, T.M., J.G.C. van de Meene, J.T. Bonner, and D.S. Barkley. 1967. The acrasin activity of adenosine-3', 5'-cyclic phosphate. Proc. Natl. Acad. Sci. 58: 1152-1154.

Mehdy, M.C., D. Ratner, R.A. Firtel. 1983. Induction and modulation fo cell-type-specific gene exppression in Dictyostelium. Cell 32: 761-771

Milne, J.L. and M.B. Coukell. 1991. A Ca ${ }^{++}$transport system associated with the plasma membrane of Dictyostelium discoideum is activated by different chemoattractant receptors. J. Cell Biol. 112: 103-110.

Oyama, M. and D.D. Blumberg. 1986. Interaction of cAMP with the cell-surface receptor induces cell-type-specific mRNA accumulation in Dictyostelium discoideum. Proc. Natl. Acad. Sci. 83: 4819-4823.

Pupillo, M., A. Kumagai, R.A. Firtel, and P.N. Devreotes. 1989. Multiple $\alpha$ subunits of guanidine nucleotide-binding proteins in Dictyostelium. Proc. Natl. Acad. Sci. 86: 48924896.

Rathi, A., S.C. Kayman, and M. Clarke. 1991. Induction of gene expression in Dictyostelium by prestarvation factor, a factor secreted by growing cells. Dev. Genet. 12: 82-87.

Saxe, C.L., R.L. Johnson, P.N. Devreotes, and A.R. Kimmel. 1991a. Expression of a cAMP receptor gene of Dictyostelium and evidence for a multigene family. Genes Develop. 5: 1-8.

. 1991b. Multiple cell-surface, receptor genes in Dictyostelium. Dev. Genet. 12: 6-13.

Schaap, P.and W. Spek. 1984. cAMP binding to the cell-surface during development of Dictyostelium discoideum. Differentiation 27: 83-87.

Schaap, P. and R. van Driel. 1985. The induction of postaggregative differentiation in Dictyostelium discoideum. Exp. Cell Res. 159: 388-398.

Shaw, D.R., H. Richter, R. Giorda, T. Omachi, and H.L. Ennis. 1989. Nucleotide sequences of Dictyostelium discoideum developmentally regulated cDNAs rich in (AAC) imply proteins that contain clusters of asparagine, glutamine, or threonine. Mol. Gen. Genet. 3: 453-459.

Siegert, F. and C.J. Weijer. 1991. Analysis of optical density wave propagation and cell movement in the cellular slime mould Dictyostelium discoideum. Physica D. 49: 224-232.

1992. Three-dimensional waves organize Dictyostelium slugs. Proc. Natl. Acad. Sci. 89: 6433-6437.

Sun, T.J. and P.N. Devreotes. 1991. Gene targeting of the aggregation stage cAMP receptor CAR1 in Dictyostelium. Genes \& Dev. 5: 572-582.

Theibert, A., M. Palmisano, B. Jastorff, and P. Devreotes. 1986 The specificity of the cAMP receptor mediating activation of adenylate cyclase in Dictyostelium discoideum. Dev. Biol. 
114: 529-533.

Traynor, D., R.H. Kessin, and J.G. Williams. 1992. Chemotactic sorting to cAMP in the multicellular stages of Dictyostel. ium development. Proc. Natl. Acad. Sci. 89: 8303-8307.

van Haastert, P.J.M. 1987. Down-regulation of cell-surface cAMP receptors and desensitization of cAMP-stimulated adenylate cyclase by cAMP in Dictyostelium discoideum. $J$. Biol. Chem. 262: 7700-7704.

van Haastert, P.J.M., M.J. de Vries, L.C. Penning, E. Roovers, J. van der Kaay, C. Erneux, and M.M. van Lookern Campagne. 1989. Chemoattractant and guanosine $5^{\prime}$-[-thio]triphosphate induce the accumulation of inositol 1,4,5-trisphosphate in Dictyostelium cells that are labeled with $\left[{ }^{3} \mathrm{H}\right]$ inositol by electroporation. Biochem J. 258: 577-585.

Williams, J.G. 1991. Spatial regulation during Dictyostelium development. Curr. Opin. Genet. Dev. 1: 358-362.

Williams, J.G., A. Ceccarelli, S.J. McRobbie, H. Mahbubani, R.R., Kay, A. Early, M. Berks, and K.A. Jermyn. 1987. Direct induction of Dictyostelium prestalk gene expression by DIF provides evidence that DIF is a morphogen. Cell 49: 185192.

Wu, L. and P.N. Devreotes. 1991. Dictyostelium transiently express eight distinct $G$ protein alpha-subunits during its developmental program. Biochem. Biophys. Res. Commun. 179: 1141-1147. 


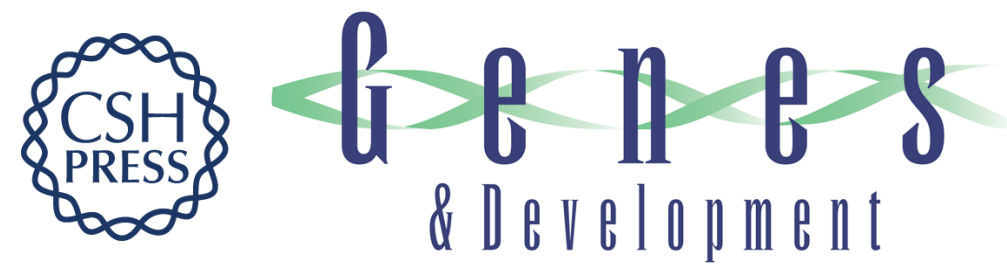

\section{CAR2, a prestalk cAMP receptor required for normal tip formation and late development of Dictyostelium discoideum.}

C L Saxe, G T Ginsburg, J M Louis, et al.

Genes Dev. 1993, 7:

Access the most recent version at doi:10.1101/gad.7.2.262

References This article cites 42 articles, 23 of which can be accessed free at:

http://genesdev.cshlp.org/content/7/2/262.full.html\#ref-list-1

License

Email Alerting

Service

Receive free email alerts when new articles cite this article - sign up in the box at the top right corner of the article or click here.

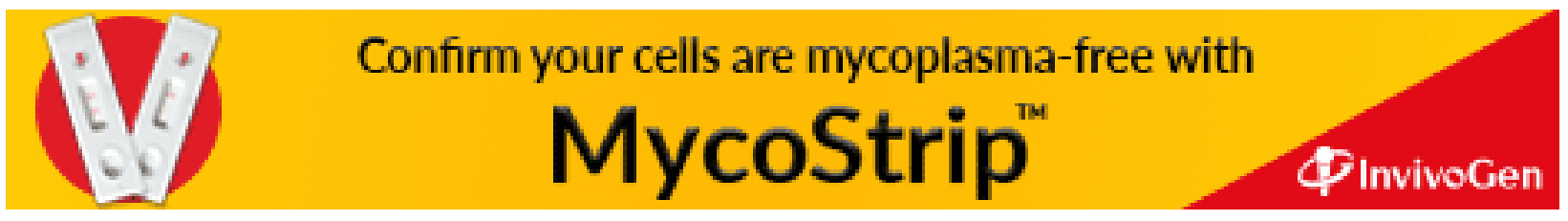

